\title{
Gas Pressure Control System for Air-assisted Extrusion Based on PLC Tian-Wen DONG ${ }^{1, a,{ }^{*}}$ and Chun-Lai TIAN² \\ ${ }^{1}$ Key Laboratory of Polymer Preparation and Processing Jiangxi Province, Shangrao Normal University, China, 334000 \\ ${ }^{2}$ School of Mechanical and Electronic Engineeing, Pingxiang University, Pingxiang, China, 337000 \\ a274629251@qq.com
}

Keywords: Gas pressure control, Air-assisted extrusion, PLC.

\begin{abstract}
A gas pressure control instrument for air-assisted extrusion has been manufactured by us. The principle, hardware connection and software design of this controller are introduced in this paper. The PLC micro controller is assembled in hard connection with pressure sensor and control valve. The $24 \mathrm{~V}$ power supply is chosen as the main control power. Software design with codes is shown through the successful experimental tests. It is obtained that the gas pressure error controlled is less $2 \%$. The design optimization with hard and soft item in the system will be carried out in the future research.
\end{abstract}

\section{Introduction}

Gas assisted extrusion is a kind of polymer processing technology [1]. In air-assisted extrusion of polymer, the controlling of the gas pressure is a very key problem. The precision of the pressure of gas directly affects the stability of extrusion process [2]. There are usually two control methods: one method is using the IPC (Industrial Personal Computer) as the control kernel, another is using the PLC (Programmable Logic Controller) [3-5]. The cost of the latter is lower and the stability is as well as the former. Thus, the PLC is selected as our control kernel.

In this paper, the principle, hardware and software of the controller is introduced. In section 1, the principle of the instrument is introduced. In section 2, the hardware connection is introduced. In section 3 , the software design is introduced.

\section{Principle of the Gas Pressure Controller}

Fig. 1 shows the gas pressure controller's principle diagram. The controller is composed of the components in the dashed line box. It includes a touch screen, a PLC with AD/DA module, a gas pressure sensor and a electromagnetic pneumatic valve. The pressure sensor is mounted in a polymer extrusion die. In the process of plastic extrusion, if the gas pressure is not controlled, it will continue to change. Before the extruder begins to work, we first open the air pump and the gas pressure controller.

The pressure value and PID parameters can be set and adjusted by touch screen. When the pressure value detected by the sensor is different from the value set by touch screen, a PID control algorithm will be calculated in PLC, which will output an analog signal through the DA module to control the opening of the electropneum valve, thus to adjust the output gas pressure. 


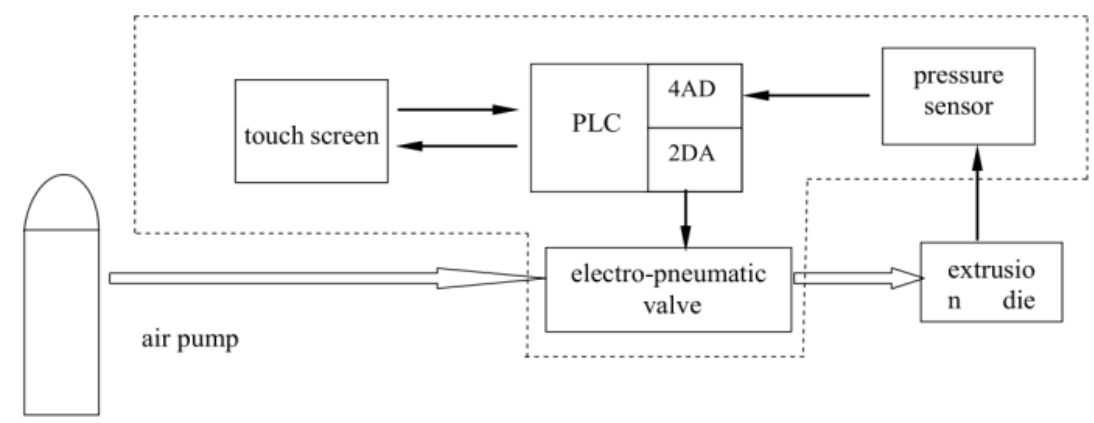

Figure 1. Gas pressure controller's principle diagram

\section{Hardware Connection of the Controller}

Fig. 2 shows the gas pressure controller's hardware connection diagram. In order to facilitate future system expansion, the FX1N-24MT PLC, FX2N-4AD and FX2N-2DA are chose. The pressure sensor and the electro-pneumatic valve separately belong to current output type and current input type. An additional $24 \mathrm{~V}$ power supply is used.

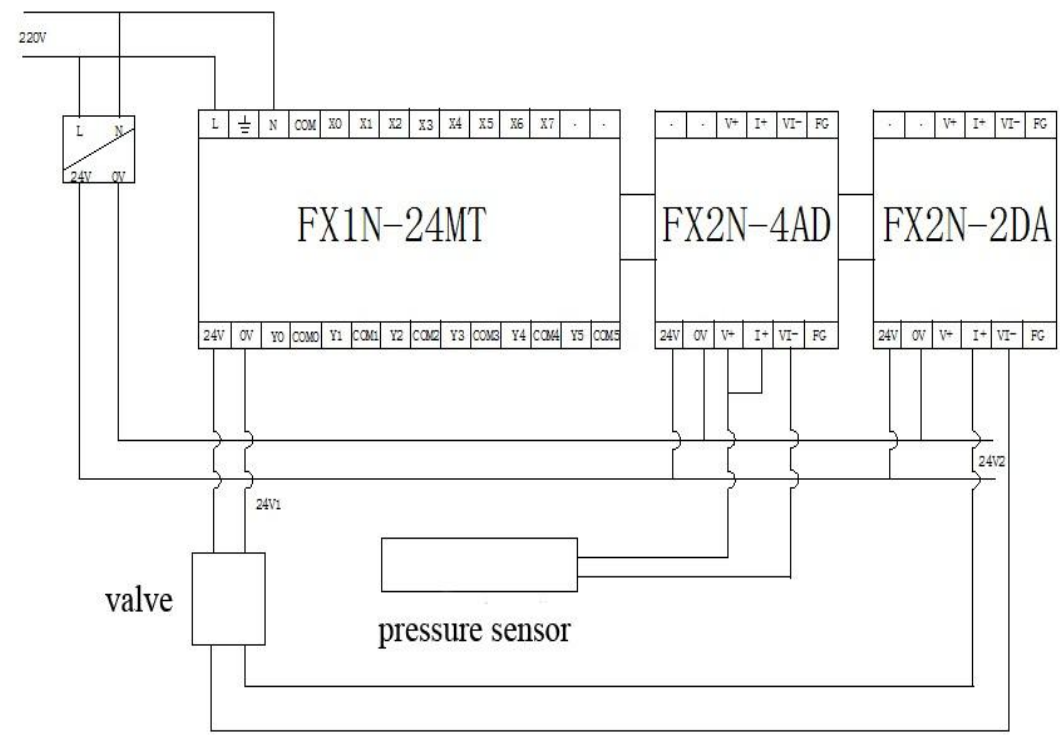

Figure 2. Hardware connection diagram

\section{Software Design of the Controller}

0 LD M8000

1 MOV D310 D200

6 MOV D300 D100

11 MOV H1 D101

16 MOV D302 D102

21 MOV D303 D103

26 MOV D304 D104

31 MOV D305 D105

36 MOV D306 D106

41 MOV K2000 D204

46 LD M8002

47 FROM K0 K30D4 K1

56 CMPK2010 D4 M0

63 LD M1 


$\begin{array}{llllll}64 & \text { TO K0 K0 H3331 K1 } & \\ 73 & \text { TO K0 K1 K4 K1 } & & \\ 82 & \text { FROM K0 K29K4M10 K1 } & \\ 91 & \text { ANI M10 } & & & \\ 92 & \text { ANI M20 } & & & \\ 93 & \text { FROM K0 K5 D202 } & \text { K1 } & \\ 102 & \text { LD M8000 } & & & \\ 103 & \text { MOV K0 D107 } & & \\ 108 & \text { PIDD200 } & \text { D202 } & \text { D100 } & \text { D204 } \\ 117 & \text { LD M8000 } & & \\ 118 & \text { MOV D204 K4M100 } & \\ 123 & \text { TO K1 K16K2M100 } & \text { K1 } & \\ 132 & \text { TO K1 K17H4 K1 } & & \\ 141 & \text { TO K1 K17H0 K1 } & & \\ 150 & \text { TO K1 K16K1M108 } & \text { K1 } & \\ 159 & \text { TO K1 K17H2 K1 } & & \\ 168 & \text { TO K1 K17H0 K1 } & & \\ 177 & \text { END } & & & \end{array}$

The codes above show the software design of the controller. The parameters set by touch screen will be saved in the data registers such as D310, D300 and others. For example, the pressure value is saved in D310 that will be sent to D200. The initial output value (2000) is saved in D204. The detected value is saved in D202. The codes from 46 to 102 implement the signal acquisition from the pressure sensor. The code 108 implement the PID control. The codes from 117 to 177 implement the DA transmit and control of electro-pneumatic valve.

\section{Summary}

A gas pressure control instrument based on PLC and touch screen is designed for the air-assisted. The extrusion experiment shows that the gas pressure error is controlled within $2 \%$.

\section{Acknowledgements}

This research was financially supported by the Industrial Support Project of Science and Technology Department of Jiangxi Province (20141BBE50014) and Science and Technology planning project of Education Department Jiangxi Province(GJJ14718).

\section{References}

1. D. R. Arda, M. R. Mackley, Sharskin instabilities and the effect of slip from gas-assisted extrusion, Rheol. Acta. 44 (2005) 352-359.

2. H. S. Liu, X. Z. Deng, Y. B. Huang et al, Effect of air pressure on interface profile in gas-assisted coextrusion process, Polymer materials science and engineering. 30(2014) 119-123.

3. C. W. Son, K. E. Choi, K. Oh , et al, Development of Gas Control System with PLC for PNU-RICH2, Journal of the Korean Physical Society. 59 (2011) 1628-1631.

4. H. Q. Liu, M. Chen, J. H. Zhu, The application of PLC in the Feed Gas Compressor Control System, Techniques of Automation \& Applications. 13(2014) A206.

5. Y. B. Cui, Y. SUN, Y. S. Tan, The design of the boiler exit flue gas temperature control system based on PLC, Machinery Design \& Manufacture. 5(2008) 83-84. 